Dhaka Univ. J. Biol. Sci. 31(1): 183-191, 2022 (January) DOI: https://doi.org/10.3329/dujbs.v31i1.57927

\title{
ADAPTATION OF THE BRAIN INJURY QUESTIONNAIRE OF SEXUALITY INTO BANGLA AND INITIAL FINDINGS FROM A SAMPLE OF BANGLADESHI TRAUMATIC BRAIN INJURY PATIENTS
}

\author{
Md. Shahanur Hossain, Graham E. Powell and \\ MOHAMmad MaHMUdur RaHMan \\ Department of Clinical Psychology, Room No. 5017, Arts Building, \\ University of Dhaka, Dhaka 1000, Bangladesh
}

Key words: Adaptation, Traumatic, Brain Injury, Sexuality

\begin{abstract}
Bangladesh is an over populated country with a high incidence of traumatic brain injury (TBI) for which neuropsychological services are only just emerging. In this context the present study undertook in a culturally sensitive manner to translate into Bangla (Bengali) the Brain Injury Questionnaire of Sexuality (BIQS) and to present findings from a sample of TBI patients. Standard procedures including back translation for scale adaptation were followed. The Bangla version of BIQS (BIQS-B) were applied on 30 TBI and 53 healthy sample. Factor and principle component analysis of the BIQS-B yielded three components as in the original scale explaining $75.3 \%$ of the total variance. Reliability is satisfactory, matching that of the English version. The TBI sample scored lower on the BIQS-B than did the healthy controls by virtue of not reporting the positive changes in sexuality over time that the health controls reported (means of 43.90 vs $52.02, \mathrm{p}<$ 0.001). In a very different culture, the factor structure of the BIQS-B is in fact similar to the English version and has acceptable reliability and validity, hence contributing to the goal of improving access to services not just in Bangladesh but in Bangla speaking minority ethnic groups in other countries.
\end{abstract}

\section{Introduction}

Traumatic Brain Injury (TBI) is considered a silent epidemic ${ }^{(1-2)}$ causing disease and disability among 69 million people per year globally(3) and projected to become the third largest cause of disease burden worldwide by 2020. Bangladesh is no exception, at least $14 \%$ of the general population having experienced at least a mild TBI and with similar sequelae to those reported in the West(4). In general, long-term changes in the cognitive, emotional, behavioral and personality spheres are common ${ }^{(5)}$ and this includes specific problems with sexuality(6). Indeed, one study suggests that significant sexual changes are experienced by more than $50 \%$ of TBI individuals up to 5 years post-injury ${ }^{(7)}$. With the

*Author for correspondence: 〈Shossain_cp@du.ac.bd>. 
advancement of medical treatment the survival rate after TBI increases and the need to manage long term sequelae rises, including the area of sexuality. An issue in a country such as Bangladesh is that sexuality is a topic often not recognized or prioritised, and can even be actively avoided. There is a reticence to talk about sexual matters and individuals may not have the language, the vocabulary, to do so; do not know how to talk about sexuality. This is where a standardised questionnaire can make a real contribution, normalising the topic and providing the words to use.

Currently no valid measure of change in sexuality exists in Bangladesh and therefore this study seeks to fill this gap by translating, adapting and validating the Brain Injury Questionnaire of Sexuality (BIQS) ${ }^{(7-8)}$. We will refer to the Bangla (Bengali) version as the BIQS-B.

\section{Objectives of the study}

1. To translate the Brain Injury Questionnaire of Sexuality (BIQS) into Bangla.

2. To determine the psychometric properties of the resultant BIQS-B, and

3. To present initial findings from a sample of Bangladeshi TBI patients.

\section{Materials and Methods}

Translation and back translation: Two bilingual psychologists who work with Acquired Brain Injury (ABI) patients translated the BIQS from English into Bangla with the kind written permission of Dr Jennie Ponsford. Since sexuality is a taboo, a hidden subject in the culture of Bangladesh, feedback on the translated version was obtained from TBI and non-TBI persons, in particular whether the wording was understandable and, importantly, whether the wording was culturally and personally acceptable.

In response to this feedback some items were rewritten and a final Bangla version along with the original English version was sent to 32 bilingual experts from related fields such as psychiatry, neurosurgery, clinical psychology, urology, endocrinology, dermatology and general physicians, with the request that they rate the validity of the translation of each of the 15 items using a 5 point Likert scale ( $1=$ not valid, $2=$ valid to some extent, $3=$ moderately valid, $4=$ mostly valid, $5=$ completely valid). Ratings were received from 22 of the experts. For each item the majority of the 22 experts $(70 \%$ to $100 \%$ ) rated that item as moderately to completely valid, and so no item changes were required.

This Bangla version of the BIQS was then back translated into English by two different experts, one a linguist from the English Department of Dhaka University and the other a bilingual clinical psychologist. Neither had previously seen the BIQS or earlier translated versions. The back translations were consolidated into one back translation by a UK based clinical neuropsychologist, a native English speaker, and this was sent to the original author of the BIQS to check for distortion. There being no 
distortion of any item according to the original author, this Bangla version, now termed the BIQS-B, was accepted for the current field testing.

\section{Field testing}

Sample and procedure: The patient sample was drawn from 62 consecutive TBI cases who had been in a relationship (approximately $65 \%$ of which was a marital relationship) at the time of the TBI and who were being seen in the neurosurgery outpatient department of Dhaka Medical College Hospital, from whom ethical permission was obtained. Patients were being followed up subsequent to an inpatient stay for TBI, or were presenting with complaints related to a previous TBI that had been managed without admission but which now seemed to have had longer term consequences. All patients were interviewed by the psychology service and given free psychoeducation and supportive counselling. Because of limitations in the content of medical records the severity of TBI was not always clear but the majority of cases, at least $65 \%$, were moderate to severe TBI by standard Mayo classification criteria. At six months post TBI each patient was contacted by telephone, offered further remote counselling at no cost to themselves and invited to participate in this study. Written or verbal consent to participate in this study was obtained. A complete data set was obtained from 30 of these patients, whose gender balance ( 27 males) and mean age (35.0 years) did not differ significantly from the original sample of 62 TBI (such a dropout rate is not unusual in a country such as Bangladesh with infrastructure issues to do with travel and communications and difficulty sustaining appointments for practical reasons rather than lack of motivation). Partial data was obtained from a further 9 patients, so although the main exploration of factor structure was based on an $\mathrm{N}$ of 30 , the maximum $\mathrm{N}$ for any individual analysis was 39.

A non clinical healthy control sample of 53 participants who had been in a relationship for at least a year was recruited via social different media (eg Facebook, WhatsApp), and data was collected online using Google Form. Due to the sensitive, personal nature of the data collected, only very limited personal demographics such as age, sex, were collected to avoid any possibility of any participant being identified. Again, consent was obtained from all participants.

Instruments: Along with the initial structured clinical interview of the patient sample, the following instruments were used:

Brain Injury Questionnaire of Sexuality, Bangla version (BIQS-B): It consists of 15 items each to be rated on a 5-point scale, and it breaks down into three subscales, sexual functioning (items 1-8), quality of relationship and self esteem (items 9-13), and mood (items 14-15). The items and short names are to be found in Appendix 1. The patient group were asked to compare current status with pre TBI status, and the control group was asked to compare current status with one year ago. 
The Relationship Assessment Scale (RAS): The RAS (9) has been adapted into Bangla (10). Used to assess relationship satisfaction in general, it is a 7 item 5 point Likert scale whereby $1=$ low satisfaction and 5 =high satisfaction. The RAS is reported as a robust scale with satisfactory psychometric properties in both English and Bangla (eg Cronbach's alpha of 0.82 and test retest reliability of $r=0.88$ in the Bangla version) The RAS has been found to relate to constructs such as love and sexual attitude (9) and romantic relationship ${ }^{(11)}$.

\section{Results and Discussion}

Factor structure: For the purpose of examining the factor structure of the BIQS-B the data from both patient and control groups were combined. The Kaiser-Mayer-Olkin value was .866 , which is higher than recommended value of $0.6^{(12)}$, and Bartlett's Test of Sphericity ${ }^{(13)}$ was significant $(\mathrm{P}<.001)$. As in the English version, principle component analysis identified three components with eigenvalues exceeding 1, explaining between them $75.3 \%$ of the total variance. The scree plot (Fig. 1) shows a clear break after the third component. Therefore, as in the English version, the total score on BIQS-B can be broken down into three subscale scores.

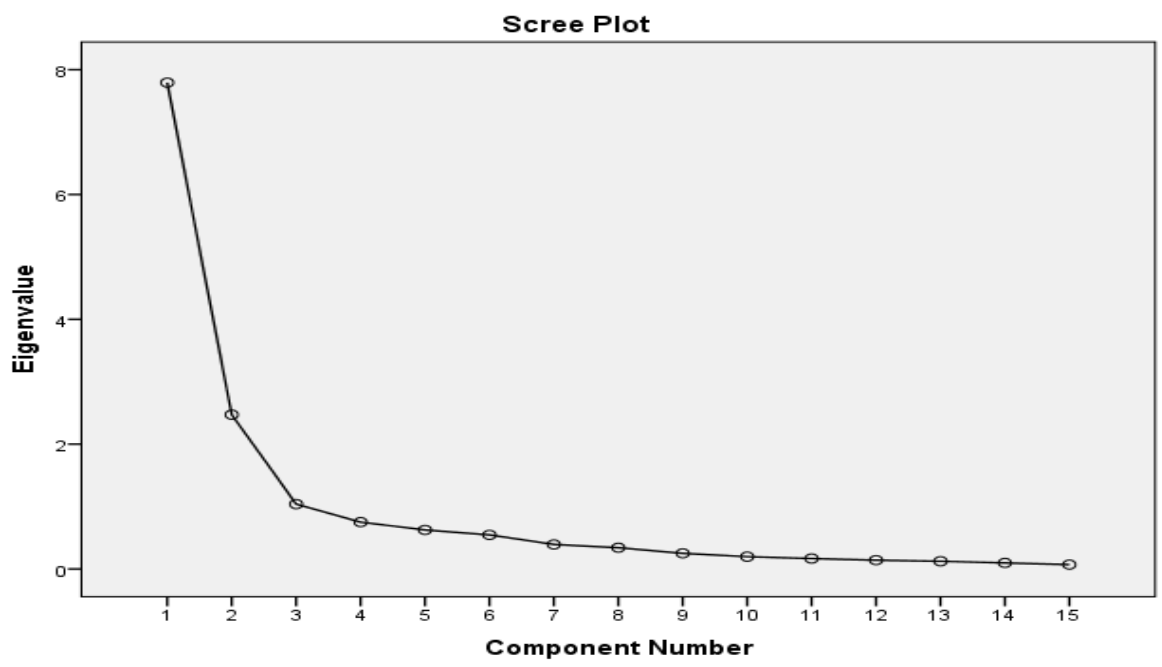

Fig. 1. Scree plot of the BIQS-B.

The three factors were similar to those obtained from the English version, in which the total score was broken down into three subscale scores, ie sexual functioning (items 18), relationship quality and self-esteem (items 9-13) and mood (items 14-15). For example, on the BIQS-B items Satisfaction, Engage, Enjoy, Aroused and Climax all loaded highly on sexual functioning, Depression and Preoccupation both loaded highly on mood, and Opportunity and Communication loaded on relationship quality and self esteem. The 
nature of the factors was not entirely identical between the BIQS and BIQS-B because items to do with self esteem on the BIQS-B (ie Confidence and Appeal) loaded on sexual functioning rather than relationship quality and self-esteem. Given the broad factorial similarities the BIQS subscale scoring was retained for the BIQS-B and for the remaining analysis as set out below.

Item analysis: The BIQS-B full scale, total score was broken down into three subscale scores as per the English version. Cronbach's alpha for full scale and sub-scale scores were satisfactory, very similar to those in the original English version (Fig. 2).

each item, between each item and the total score, and between each item and its appropriate subscale score. (All correlations were significant, as would be expected from the high Cronbach's alphas, and so precise significance levels for each correlation will not be given).

To examine inter item consistency, correlation coefficients were computed between

Each item correlated significantly with the total score. The lowest correlation was for item 9, Opportunity, which is understandable as it is an item that relates to environmental circumstances not under the control of the individual.

Correlations between items 1-8 and the total score ranged from .755 to .870 . Inter item correlations for items 1-8 ranged from .501 to .885 . Correlations with the subscale score ranged from .755 to .865 .

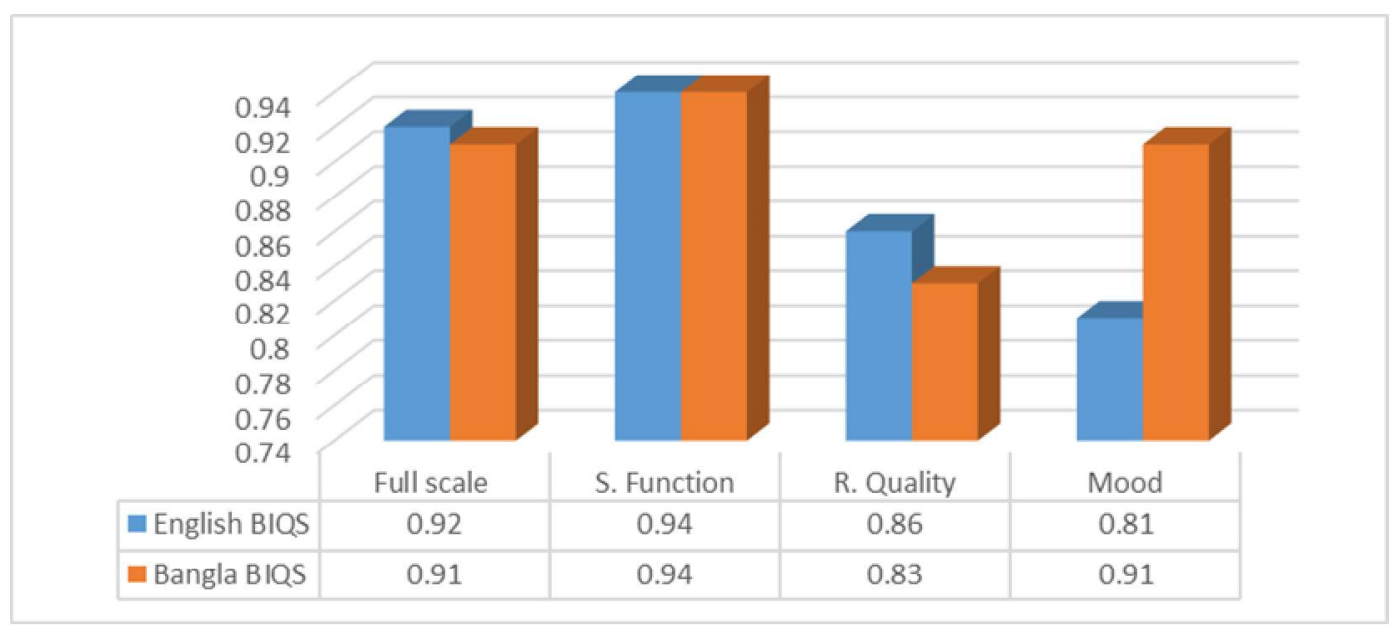

Fig. 2. Cronbach's Alpha for the BIQS and BIQS-B.

Correlations between items 9-13 and the total score ranged from .229 (for item 9 as discussed above) to .815. Inter item correlations for items 9-13 ranged from .207 (because of item 9) to .827 . Correlations with the subscale score ranged from .420 to .765. The correlations between items $14-15$ and the total BIQS-B score were .384 and .396 
respectively. Inter item correlation was .841. Item correlations with the mood subscale score were both .841 .

Convergent validity: Correlations were computed between the total and sub-scale scores of the BIQS-B and the total RAS score. Total BIQS-B score correlated $.37(\mathrm{p}<02)$ with total RAS score. Relationship quality and self-esteem and mood both correlated significantly with the RAS ( $\mathrm{r}=.48$ and .52 respectively, $\mathrm{p}<01$ ), but sexual functioning did not correlate significantly with the RAS $(\mathrm{r}=.15, \mathrm{p}=.21, n s)$. This in itself is an interesting finding, that in Bangladesh sexual functioning and relationship satisfaction are not correlated. The quality of the relationship is not dependent on the quality of sexual functioning.

Discriminant validity: Total and subscale scores on the BIQS-B of the TBI and control groups were compared using ANOVA. Mean scores of patients on BIQS-B were all lower than for mean scores of the control group, whereas there was no significant difference on the RAS (Table 1)

Table 1. Mean scores on the BIQS-B and RAS for patients and controls.

\begin{tabular}{lccccc}
\hline Variables & Sample & $\mathrm{N}$ & Mean & $F$ & $P$ difference \\
\hline \multirow{2}{*}{ BIQS Total } & TBI & 30 & 43.90 & \multirow{2}{*}{13.50} & $<.001$ \\
& NC & 53 & 52.02 & & \\
BIQS Sexual Function & TBI & 34 & 24.32 & 7.76 & .007 \\
BIQS Relationship quality & NC & 53 & 28.40 & & \\
and self esteem & TBI & 32 & 14.38 & 7.96 & .006 \\
BIQS Mood & NC & 53 & 16.81 & & \\
& TBI & 39 & 5.41 & \multirow{2}{*}{9.93} & .002 \\
RAS Total & NC & 53 & 6.81 & & \multirow{2}{*}{ n.s. } \\
\hline
\end{tabular}

The fact that the TBI group scores lower on the BIQS-B than does the control group does not mean that the patients see a significant deterioration in sexuality. The midpoint of 3 on any item indicates no change, therefore a score of 45 on the total BIQS-B would mean no change, and similarly a score of 24 on sexual function would indicate no change, 15 on relationship quality and self esteem would indicate no change, and 6 on mood would indicate no change. As can be seen from table 1, the means for the TBI group are very similar indeed to these 'no change' scores. The overall pattern, then, is that the TBI group sees no change since the TBI whereas the control group reports an improvement in sexuality across time. 


\section{Conclusions}

In a very different culture, one in which there are particular sensitivities with regard to discussing intimate matters, the factor structure of the BIQS-B is in fact similar to the English version and has acceptable reliability and validity for the measurement of sexual functioning, quality of relationship and self-esteem and mood as related to sexuality, hence potentially contributing to the goal of improving access to services not just in Bangladesh but in Bangla speaking minority ethnic groups in other countries. The BIQS$B$ will clinically assist with identifying issues and the measurement of progress.

An unexpected finding of the study was that the TBI group did not report any overall deterioration in sexuality on the BIQS-B, so the clinical need for an intervention will depend on the individual case. However, the mean report of no change might reflect to some extent the TBI itself, might arise from deficits attributable to that TBI, especially as the majority of the cases were in the moderate to severe category. There are potentially at least two mechanisms by which this might in part occur. First, the report of no change could reflect a relative lack of insight on the part of at least some of the TBI group, so it will be important in the future to look at the partner's report of sexual changes. Second, there was be a subgroup of TBI cases who reported an improvement in sexuality but in whom such an improvement is in fact the expression of disinhibition, a reflection of a frontal lobe or dysexecutive syndrome. This sort of findings for both groups are to be found in Appendix 2. Again, this suggests that the interpretation of BIQS-B responses should be undertaken on a case by case basis in the context of reports from significant others and a broader analysis of neuropsychological profile.

As the results stand, though, the picture is clinically quite positive for the Bangladeshi context; sexuality is quite resilient to TBI and the quality of relationships does not arise purely from issues of sexual functioning.

\section{Acknowledgement}

This project is funded by the Neuroscience Research Center of the University of Dhaka, Dhaka-1000, Bangladesh.

\section{References}

1. Rusnak M 2013. Traumatic brain injury: giving voice to a silent epidemic. Nat Rev Neurol. 9: 186-187.

2. Vaishnavi S, V Rao and JR Fann 2009. Neuropsychiatric problems after traumatic brain injury: unraveling the silent epidemic. Psychosomatics 50: 198-205.

3. Dewan MC, A Rattani, S Gupta, RE Baticulon, Y Hung, M Punchak, A Agrawal, AO Adeleye, MG Shrime, AM Rubiano, JV Rosenfeld, and KB Park 2018. Estimating the global incidence of Traumatic Brain Injury. Journal of Neurosurgery, Published online April 27, 2018; DOI: 10.3171/2017.10.JNS17352. 
4. Hossain MS, N Khatun, KU Chowdhury, GE Powell, R McCarter and N Walton 2016. Prevalence of post concussion symptoms in an Asian country; base rates and effects of mild traumatic brain injury. Paper presented at the Eleventh world congress on brain injury, The Hague, Netherland.

5. Martin LT, C Farris, AM Parker and C Epley 2013. The Defense and Veterans Brain Injury Center Care Coordination Program: Assessment of Program Structure, Activities, and Implementation. Rand health quarterly 3(3): 4 .

6. Calabrò RS, M Russo and A Naro 2017. Discussing Sexual Health after Traumatic Brain Injury: An Unmet Need! Innovations in clinical neuroscience 14(1-2): 11-12.

7. Ponsford J 2003. Sexual changes associated with traumatic brain injury. Neuropsychological rehabilitation. 13. 275-89. 10.1080/09602010244000363.

8. Stolwyk RJ, MG Downing, J Taffe, JS Kreutzer, N Zasler and J Ponsford 2013. Assessment of sexuality following traumatic brain injury: validation of the Brain Injury Questionnaire of Sexuality (BIQS). J Head Trauma Rehabil. 28: 164-170.

9. Hendrick SS 1988. A generic measure of relationship satisfaction. Journal of Marriage and the Family, 93-98

10. Chowdhury T, MA Islam and T Rahman 2016. Adaptation of Relationship Assessment Scale for use in Bangladesh. Bangladesh Psychological Studies 26: 25-32.

11. Hendrick SS, A Dicke, and C Hendrick 1998. The relationship assessment scale. Journal of Social and Personal Relationship 15(1): 137-142.

12. Kaiser H 1970. A second generation Little Jiffy. Psychometrika 35: 401-415.

13. Bartlett MS 1954. A note on the multiplying factors for various chi square approximations. Journal of the Royal Statistical Society 16 (Series B): 296-298.

(Manuscript received on 4 August, 2021; accepted on 25 November, 2021) 
Appendix 1. Brain injury questionnaire of sexuality items and short names.

\begin{tabular}{lll}
\hline 1 & The importance of sexuality & Importance \\
2 & Your sex drive & Drive \\
3 & The frequency with which you engage in sex & Frequency \\
4 & Your ability to give your partner sexual & Satisfaction \\
& satisfaction/enjoyment/fulfilment & \\
5 & Your ability to engage in intercourse & Engage \\
6 & Your ability to enjoy sex yourself & Enjoy \\
7 & Your ability to stay aroused during sex & Aroused \\
8 & Your ability to climax & Climax \\
9 & Your opportunity to engage in sex & Opportunity \\
10 & Your ability to verbally communicate with your sexual partner & Communication \\
11 & Your self-confidence & Confidence \\
12 & Your sex appeal & Appeal \\
13 & The quality of your relationship with your sexual partner & Relationship \\
14 & Level of depression & Depression \\
15 & Level of preoccupation with problems & Preoccupation \\
\hline
\end{tabular}

Appendix 2. Component of sexual functioning: comparison between TBI and NC groups.

\begin{tabular}{|c|c|c|c|c|c|}
\hline $\begin{array}{l}\text { Component of sexual } \\
\text { functioning }\end{array}$ & Response & $\begin{array}{l}\text { TBI group } \\
\text { (n) }\end{array}$ & $\begin{array}{l}\text { NC group } \\
\text { (n) }\end{array}$ & $\begin{array}{c}\text { Chi } \\
\text { square }\end{array}$ & $P$ \\
\hline \multirow{3}{*}{ Importance of sexuality } & Increased & 16 & 22 & \multirow{3}{*}{2.02} & \multirow{3}{*}{.363} \\
\hline & No change & 17 & 26 & & \\
\hline & Decreased & 8 & 5 & & \\
\hline \multirow{3}{*}{ Sex Drive } & Increased & 18 & 22 & \multirow{3}{*}{3.80} & \multirow{3}{*}{.149} \\
\hline & No change & 16 & 28 & & \\
\hline & Decreased & 7 & 3 & & \\
\hline \multirow{3}{*}{ Frequency } & Increased & 15 & 18 & \multirow{3}{*}{1.15} & \multirow{3}{*}{.563} \\
\hline & No change & 12 & 22 & & \\
\hline & Decreased & 12 & 13 & & \\
\hline \multirow{3}{*}{ Partner Satisfaction } & Increased & 11 & 27 & \multirow{3}{*}{4.86} & \multirow{3}{*}{.088} \\
\hline & No change & 18 & 23 & & \\
\hline & Decreased & 6 & 3 & & \\
\hline \multirow{3}{*}{ Engagement } & Increased & 14 & 28 & \multirow{3}{*}{10.97} & \multirow{3}{*}{.004} \\
\hline & No change & 15 & 23 & & \\
\hline & Decreased & 11 & 2 & & \\
\hline \multirow{3}{*}{ Enjoyment } & Increased & 14 & 27 & \multirow{3}{*}{4.37} & \multirow{3}{*}{.112} \\
\hline & No change & 19 & 23 & & \\
\hline & Decreased & 7 & 3 & & \\
\hline \multirow{3}{*}{ Arousal maintenance } & Increased & 12 & 28 & \multirow{3}{*}{8.92} & \multirow{3}{*}{.012} \\
\hline & No change & 18 & 22 & & \\
\hline & Decreased & 10 & 3 & & \\
\hline \multirow{3}{*}{ Climax } & Increased & 13 & 29 & \multirow{3}{*}{5.19} & \multirow{3}{*}{.074} \\
\hline & No change & 20 & 20 & & \\
\hline & Decreased & 7 & 7 & & \\
\hline
\end{tabular}

